\title{
Postpartum Depression Relating to Smoking and Drinking Habits of Husbands Among Rajbanshi Mothers in Nepal
}

\author{
Nawa Raj Subba*, Shishir Subba \\ Tribhuvan University, Kathmandu, Nepal \\ Email address: \\ dr.subba2015@gmail.com (N. R. Subba), shishirsubba21@gmail.com (S.Subba)
}

To cite this article:

Nawa Raj Subba, Shishir Subba. Postpartum Depression Relating to Smoking and Drinking Habits of Husbands Among Rajbanshi Mothers in Nepal. American Journal of Health Research. Vol. 3, No. 5, 2015, pp. 293-297.doi: 10.11648/j.ajhr.20150305.15

\begin{abstract}
This is a cross-sectional study on postpartum depression (PPD) among Rajbanshi mothers in Nepal. The objective of the study was to find out the prevalence rate of PPD and association between PPD in mothers with smoking/drinking habits of the husbands and perceived stress among Rajbanshi community. Quantitative methods employed using semi-structured questionnaires. The sample size was 375 households from Morang, Jhapa and Sunsari districts. Data collected by in-depth interviews with mothers. Researcher filled the semi-structured questionnaires asked on workload, sleep, perceived stress and treatment history using the Edinburgh Postnatal Depression Scale during interviews. In the findings, PPD and maternal stress associate with husband's smoking/drinking habits. Prevalence of PPD was $12.27 \%$ in sampled Rajbanshi mothers (EPDS cutoff $\geq 13)$. PPD in mothers find associated with maternal stress $(\mathrm{P}=<.0001)$ and smoking habit of the husband $(\mathrm{P}=<.0001)$ which are statistically highly significant. Similarly, sleeplessness in mothers associated with smoking $(\mathrm{P}=<.005)$ and drinking $(\mathrm{P}=<.0001)$ habits of husbands are also statistically highly significant. In conclusion, PPD among Rajbanshi mothers associates with a smoking habit of the husband. Both smoking and drinking habits of husbands were responsible for causing maternal stress and sleeplessness in mothers and these factors associated with PPD. Smoking and drinking habits of husbands find out risk factors for PPD and perceived stress in her wife. To deal successfully with the poor situation of PPD among Rajbanshi mothers need to change smoking/drinking habits of husbands, raise awareness on health as well as the socio-economic development of the community.
\end{abstract}

Keywords: Postpartum Depression, Smoking, Drinking, Stress, Sleeplessness, Rajbanshi Nepal

\section{Introduction}

Nepal has multiethnic, multicultural, pluralistic society. It has enormous socioeconomic disparities, remained in politically unstable, economically low economic growth under conflict situation for long. The number of mental health disorder cases, including suicides is increasing in the country. It is urgent to give effective and affordable mental health services. Awareness about women's health, mental health along with postpartum depression is poor in the community. Psychological disturbances can manifest and seriously threaten safe and normal motherhood. Mental health is an important part of public health. Nepal government has given mental health less priority. Traditional healing practices exist and indigenous people and remote people rely on this. Rajbanshi is indigenous and considered as disadvantaged community. It has unique indigenous culture, traditions, and custom related to health care. Now they have adopted duel traditional and modern health care practices. Very few studies have done on health of Rajbanshi mothers. Study on PPD among Rajbanshi mothers in Nepal lacks in literature. This study has attempted to explore Rajbanshi PPD mothers whether any association with husbands' smoking and drinking habits.

Postpartum depression is the most common complication of childbearing, occurring in $10-15 \%$ of women after delivery has mentioned by O'Hara, et al. [1]. Demyttenaere, et al indicated that in developing countries, more than three-quarters of people with serious mental disease do not receive any treatment [2]. During their reproductive years, women are at increased risk of most disorders that affect the emotions. Holden indicated these include depression, anxiety, post traumatic stress disorder and anorexia [3]. During first delivery mothers have a more than twofold risk of needing mental health care during the first months after delivery as compared to a year later, and the increased risk of depression lasts the first five postnatal months said Munk-Olsen, et 
al.[4]. During postnatal period, depression contributes to several problems in the person, family and society. In severe depression, especially with psychotic symptoms, there is even a risk of suicide warned by Oates, [5].

\section{Review of Literature}

Based on the 143 studies from 40 countries Uriel H. [6] noted that mean of the global burden of PPD is now considered $10-15 \%$, but there is wide variation from $0 \%$ to $60 \%$. Countries like Singapore, Malaysia, Austria, Malta and Denmark there are few reports of PPD where other countries Brazil, Guyana, Costa Rica, Chile, South Africa, Taiwan and Korea reported postpartum depression very high. Socio-economic environments such as poverty, level of social support and its perception, nutrition, stress, stigma, and biological vulnerabilities caused of PPD in the countries. In China, Ho-Yen S et al [7] find out prevalence rate of PPD was $15.5 \%$ and associated with lower-income, difficult pregnancy experience, poor infant health, not attending childbirth classes and low spousal involvement before and after delivery. The study suggested that mothers who perceived unhelpful caregiver mothers had twice the odds of PPD than helpful caregiver mothers. In Goa of India, a study done by Patel et al [8] find out the prevalence rate of PPD was $23 \%$ of the mothers after 6-8 weeks after births. Major factors for illnesses recorded substantial psychological morbidity, economic deprivation, poor marital relationship and gender of the infant. The study recommended interventions to target mothers in the ante-natal period and join a strong gender-based part since depressed mothers were more disabled and m ore unlikely to use health services.

In Nepal a study done in Kathmandu Medical college and Dhading district hospital by Budhathoki et al [9] has found that incidence of postpartum depression was $19.4 \%$ and also incidence of physical, psychological and sexual violence were found. That study could not find any significant association among any forms of violence. Another study conducted in Lalitpur district Nepal by Ellen Y. et al [10] postpartum depression among mothers was strongly associated with husband's alcoholism, polygamy and earlier depression. This study also finds significant factors for PPD were stress, multiparity, smoking and traditional family structure.

\section{Objective of the Study}

The objective of the study was to find out the prevalence rate of PPD and association with perceived stress, sleep and smoking/drinking habits of the husbands among Rajbanshi mothers.

\section{Methods and Materials}

\subsection{Study Setting and Sampling}

The study employed cluster-based quantitative cross-sectional design among Rajbanshi mothers at households in three districts of Eastern Nepal, Jhapa, Morang and Sunsari. General information was taken from the head of household and detailed information taken from interviewing mothers on the semi-structured questionnaire. Probability sampling method used to decide a number of household surveys. A survey [6] showed the proportion of home delivery in eastern Terai of Nepal was $81 \%$. Therefore, Probability of home delivery being selected $\mathrm{p}=81$, and not being selected $\mathrm{q}=$ 19. According to formula, required Sample size $(n)=4 p q / L 2$ Probability of being selected in sample is (95\%) Hence,

$$
\begin{gathered}
\mathrm{L}=5 \% \text { of p. }=81 \times 5 / 100=4.05 \\
\mathrm{n}=4 \mathrm{pq} / \mathrm{L} 2=4 \times 81 \times 19 / 4.052=6156 / 16.4=375
\end{gathered}
$$

The sampling size was 375 households in the study.

\subsection{Data Collection Tools and Procedure}

The semi-structured questionnaire used to collect overall data from Rajbanshi communities, which are scattered and unevenly distributed population in 2013. A roster with the name of heads of households made. 375 households were selected as a sample by random sampling method. The tool Edinburgh Postnatal Depression Scale (EPDS) also used to find postpartum depression in mothers by asking 10 questions on clinical symptoms of depression such as any guilt feeling, sleep disturbance, low energy, anhedonia, and suicidal ideation to get scores. Screening of PPD conducted using the EPDS tool during interviews with mothers. Mothers with scale scoring 13 or more screened of possible PPD in the field. Tools were filled based on history taking with mothers. A psychiatrist and medical officers were involved as consultants in the study.

\subsection{Data Analysis}

Data collected from the study areas entered into the computer. Software SPSS-20 used for data analysis. The findings presented figures.

\subsection{Ethical Consideration}

Respondents were mothers and head of the family gave verbal consent during the interview. They ensured about information would be kept confidential. Research Committee of Tribhuvan University Institute of Humanities and Social Sciences has given permission for the study.

\section{Results}

\subsection{Demographic Factors}

Table No. 1 shows that prevalence of postpartum depression was $12.3 \%$ among Rajbanshi mothers. Of the total 46 PPD mothers (EPDS cutoff $\geq 13$ ) by economic condition 37 $(80.43 \%)$ were very poor, $4(8.7 \%)$ were poor and $5(10.87 \%)$ were rich. By education, 21 (45.65\%) were illiterate, 11 (23.91\%) were not- schooling literate, 12 (26.09\%) were class $1-10$, and $2(4.35 \%)$ were SLC/IA in education. No 
mothers recorded from BA and above among PPD. Similarly, by occupation, Labor 30 (65.22\%), agriculture 12 (26.09\%), business $2(4.35 \%)$ and service $2(4.35 \%)$. By practice, 34 (73.91\%) mothers had traditional practice or home delivery and $12(26.09 \%)$ had modern practice or hospital delivery during last baby birth.

Table 1. Demographic profile of mothers suffering from PPD.

\begin{tabular}{lll}
\hline Variable & Frequency & Percentage \\
\hline Economic & 37 & $80.43 \%$ \\
Very Poor & 4 & $8.70 \%$ \\
Poor & 5 & $10.87 \%$ \\
Rich & & \\
Education & 21 & $45.65 \%$ \\
Illiterate & 11 & $23.91 \%$ \\
Non-schooling literate & 12 & $26.09 \%$ \\
Class 1-10 & 2 & $4.35 \%$ \\
SLC & 0 & 0 \\
BA and above & & \\
Occupation & 12 & $26.09 \%$ \\
Agriculture & 2 & $4.35 \%$ \\
Service & 30 & 65.22 \\
Labour & 2 & $4.35 \%$ \\
Business & & \\
Practice & 34 & $73.91 \%$ \\
Traditional & 12 & $26.09 \%$ \\
Modern & 375 & \\
Total Respondents & 46 & $12.27 \%$ \\
PPD mothers & &
\end{tabular}

\subsection{PPD, Maternal Stress and Husband's Smoking/Drinking}

Table No. 2 shows $\chi 2$ test of association between PPD in a mother with their husbands' smoking habit. Of 46 PPD mothers $35(76.09 \%)$ husbands were smokers and 11 $(23.91 \%)$ were no smoker. It is statistically highly significant $(\mathrm{P}=<.0001)$. At the same time, 41 (89.13\%) husbands drink alcohol and $5(10.87 \%)$ did not. Hence, drinking habit of husband and PPD in wife find statistically not significant $(p=0.076) .42 \quad(91.30 \%)$ mothers had reported mental stress and $4(8.70 \%)$ did not $(\mathrm{P}=<.0001)$. There was an association between PPD and sleeplessness in mothers. 34 PPD mothers reported sleepless and 12 did not $(\mathrm{p}=<0.0001)$.

Table 2. Factors associated with PPD in mothers.

\begin{tabular}{|c|c|c|c|c|}
\hline \multirow[t]{2}{*}{ Variable } & \multicolumn{2}{|c|}{ PPD occurrence } & \multirow[t]{2}{*}{$\chi^{2}$} & \multirow[t]{2}{*}{ P-Value } \\
\hline & Yes & No & & \\
\hline \multicolumn{5}{|c|}{ Husband's habit } \\
\hline Smoker & 35 & 144 & 16.90 & $<.0001$ \\
\hline Non-smoker & 11 & 185 & d.f. $=1$ & \\
\hline \multicolumn{5}{|c|}{ Husband consume } \\
\hline Alcohol & 41 & 256 & 3.14 & 0.0764 \\
\hline No Alcohol & 5 & 73 & d.f. $=1$ & \\
\hline \multicolumn{5}{|c|}{ Maternal stress } \\
\hline Reported & 42 & 35 & 160.94 & $<.0001$ \\
\hline Not-reported & 4 & 294 & d.f. $=1$ & \\
\hline
\end{tabular}

\subsection{Maternal Sleep and Husband's Smoking/Drinking Habits}

Table No. 3 indicates $\chi 2$ test of association between sleeplessness in a mother with a smoking habit of the husband. Of 58 sleepless mothers, 38 had smoker husband and 20 had non-smoker which was statistically significant $(\mathrm{P}=<.005)$. Similarly, 52 mothers had an alcohol consuming husband and 6 not drinking husband, which was also statistically highly significant $(\mathrm{P}=<.0001)$.

Table 3. Factors associated with Sleeplessness with mothers.

\begin{tabular}{|c|c|c|c|c|}
\hline \multirow[t]{2}{*}{ Variable } & \multicolumn{2}{|c|}{ Maternal sleepless } & \multirow[t]{2}{*}{$\chi^{2}$} & \multirow[t]{2}{*}{ P-Value } \\
\hline & Yes & No & & \\
\hline \multicolumn{5}{|c|}{ Husband's Habit } \\
\hline Smoker & 38 & 20 & 8.7 & $<.005$ \\
\hline Non-smoker & 141 & 176 & d.f. $=1$ & \\
\hline \multicolumn{5}{|c|}{ Husband Consume } \\
\hline Alcohol & 52 & 6 & 94.68 & $<.0001$ \\
\hline No Alcohol & 5 & 73 & d.f. $=1$ & \\
\hline
\end{tabular}

\subsection{Risk Estimate of PPD with Smoking, Drinking, Stress and Sleep}

Table No. 4 shows bi-variate logistic regression analysis between smoking, alcohol, stress and PPD. Smoking of husband was risk for PPD in wife (OR: 3.48; CI: 1.83-6.65). Drinking by husband was also risk for stress in wife (OR: 1.76; CI: 0.95-3.26). The perceived maternal stress was significantly risk for her PPD (OR: 40.64; CI: 15.03-109.86).

Table 4. Risk Estimate.

\begin{tabular}{llllll}
\hline & & & & \multicolumn{2}{c}{$\mathbf{9 5 \%}$ Confidence } \\
\hline Variables & & & Odds Ratio & Lower & Upper \\
& Yes (\%) & No (\%) & & & \\
Smoking & PPD & & & & \\
No & 5.6 & 94.4 & & & \\
Yes & 19.6 & 80.4 & 3.48 & & \\
Drinking & Stress & & & & \\
No & 12.8 & 87.2 & & & \\
Yes & 77.4 & 22.6 & 1.76 & & \\
Stress & PPD & & & & \\
No & 1.3 & 98.7 & & & \\
Yes & 54.5 & 45.5 & 40.64 & 15.03 & 109.86 \\
\hline
\end{tabular}

\section{Discussion}

This study identified the prevalence rate of PPD was $12.3 \%$ among Rajbanshi mothers. An another study conducted in a hospital in Kathmandu finds the prevalence of PPD 19.4\% and $91.3 \%$ reported mental stress, only $8.7 \%$ reported no mental stress[7]. In another comparative study of Nepal and Norway finds out the prevalence of depressive symptoms $4.9 \%$ in Nepal and $16.5 \%$ in Norway [11].

This study identified the significant association in the sleep of mothers with husband's smoking $(\mathrm{P}=<.005, \mathrm{OR}=3.48)$ and drinking $(\mathrm{P}=<.0001, \mathrm{OR}=1.76)$ among Rajbanshi. PPD associated with maternal stress with highly significant $(<.0001)$ 
in which risk is quite high $(\mathrm{OR}=40.64)$ among Rajbansshi in Nepal. In Japan a study [12] identified sleeplessness in mothers was also associated with the husband's habit of taking alcohol was statistically highly significant. Out of 58 sleepless mothers $51(87.93 \%)$ was suffering from mental stress was also statistically highly significant. Out of 58 sleepless mothers $34(58.62 \%)$ were suffering from PPD. Therefore, PPD associated with sleeplessness was statistically highly significant. In another study conducted in Nepal has found depressive symptoms in the postnatal period were strongly associated with the woman's relationship with her husband, reflected through the custom of polygamy and the husbands' alcoholism. Postnatal depressive symptoms were also associated with previous depression, with experience mental stressful life events the previous year, depression during pregnancy, multiparity and smoking [13]. Partner alcohol problems pose diverse health threats for women. Women whose partners had alcohol problems were more likely to experience victimization, injury, mood disorders, anxiety disorders, and being in fair or poor health than women whose partners did not have alcohol problems (odds ratio: 1.7-4.5) [14]. They also experienced more life stressors and had lower mental/psychological quality-of-life scores.

In this study on Rajbanshi, mothers identified a strong association between PPD in mothers and husbands' smoking habit. Another study done in Japan has identified an association between PPD and smoking of mother self [15]. The findings of study done on Rajbanshi mothers have pushed researcher to explore literature on smoking and personality. The relationship between smoking and personality characteristics has carried out according to the theoretical model [16]. According to this model, there are three predominant dimensions of character or personality related to smoking: extroversion, neuroticism and psychoticism. It has been hypothesized that there is a relationship between extroversion and smoking. The extroversion dimension comprises factors such as sociability, assertiveness, positive emotions, vivacity, and activity level [17]. Similarly, traces of neuroticism can make the smoker sensitive to the properties of nicotine. People who get high scores on personality tests that evaluate this dimension possibly receive greater reinforcement in stressful situations, due to the stress-reducing effects provided by the cigarette. The neuroticism dimension comprises sub-dimensions of personality, such as anxiety, depression, psychological vulnerability, hostility, and anger and related to depression and anxiety disorders.

Rajbanshi mothers are getting family and social support and recognition by culture. Rajbanshi culture provides the mother with someone to take care for neonatal and older children and do their household duties. Different levels of social supports seen in different cultures. Low social support or social isolation found is risk factors for developing depression in the postnatal period [18, 19, and 20]. Therefore, traditional family support and support structures can play the role in a mother's health. Likewise, Functional help to the mother also relieves their normal workload. There is also social recognition of her new role and status. In the cultures, Stern and Kruckman suggested; there was a great deal of personal attention given to the mother. However, the literature generally found in China and Nepal shows very little attention being paid to the pregnancy; much more attention has focused on the mother after the baby is born [21].

\section{Limitation of the Study}

Literally, Rajbanshi includes various types of communities in Nepal. There is largely cultural diversity from place to place among Rajbanshi called communities and people. But the scope of study is Rajnbanshi communities living in Morang, Jhapa and Sunsari in Nepal who are Hindu by culture.

\section{Conclusion}

PPD among Rajbanshi mothers relates with a smoking habit of the husband. Smoking and drinking habits of husbands were responsible for causing maternal stress and sleeplessness in mothers and these factors associated with PPD. The study finds out the prevalence rate of postpartum depression, $12.3 \%$ among Rajbanshi mothers in Nepal. PPD in mothers find associated with maternal stress $(\mathrm{P}=<0.0001)$ and smoking habit of the husband $(\mathrm{P}=<.0001)$ which are statistically highly significant. Similarly, sleeplessness in mothers associated with smoking $(\mathrm{P}=.0032)$ and drinking $(\mathrm{P}=<0.0001)$ habits of husbands are also statistically highly significant. Smoking and drinking habits of husbands are risky habits for PPD and perceived stress in her wife.

In order to lower prevalence of PPD and mental health problems in mothers they need to have change the husband's smoking/drinking habits, raise awareness on reproductive health and rights, empowerment of mothers, enforce traditional family support, along with the strengthening of their socio-economic condition.

\section{Abbreviations}

$\begin{array}{ll}\text { ANC } & \text { Ante Natal Care } \\ \text { EDPS } & \text { Edinburgh Postnatal Depression Scale } \\ \text { PPD } & \text { Post Partum Depression }\end{array}$

\section{Authors' Contributions}

Nawa Raj Subba has played a great role with regard to the conception, design, data collection, analysis, interpretation and wrote the draft manuscript. Shishir Subba has made a contribution of study design, developed the research protocol, analysis, interpretation, and reviewed the draft. All authors read and approved the last version of the manuscript.

\section{Authors' Information}

Nawa Raj Subba has Doctor of Philosophy in Health Psychology from Tribhuvan University Nepal. He has worked more than three decades for Ministry of Healthand now working as a consultant in universities after retirement. 
Shishir Subba has Doctor of Philosophy in Psychology from The University of Copenhagen and working. He is professor in Tribhuvan University, Central Department of Psychology, Kathmandu, Nepal.

\section{Acknowledgements}

We are very grateful to Dr. Naba Raj Koirala, Dr. Sharad Baral, Dr. Krishna Shah, Dr. Bibek Subedi, and Dr. Madhur Bhagat for technical support. We also thank Jyotish Rajbanshi, Muluk Chand Rajbanshi, and Kamal Singh Rajbanshi for facilitating interviews during data collection.

\section{References}

[1] O'Hara, M. W. \& Swain, A. M. Rates and risk of postpartum depression-a meta-analysis. International Review of Psychiatry. $1999 ; 8,37-54$.

[2] Demyttenaere K, et al. Prevalence, severity, and unmet need for treatment of mental disorders in the World Health Organization World Mental Health Surveys. Jama. 2004; 1(21):2581-90.

[3] Holden, C. Sex and the suffering brain. Science. 2005; 308(5728): pp1574.

[4] Munk-Olsen et al. Postpartum Depression A Major Public Health Problem, The Journal of Americal Medical Association, JAMA. 2006; 296(21):2616-2618. doi:10.1001/jama. 2006; 296.21. 2616

[5] Oates, M. Perinatal psychiatric disorders: a leading cause of maternal morbidity and mortality. British Medical Bulletin. 2003; 67:219-29.

[6] Uriel H., Sandhya K., Cross-cultural and social diversity of prevalence of postpartum depression and depressive symptoms. Journal of Affective Disorders. April 2006Volume 91, Issues 2-3, Pages 97-111.

[7] Ho-Yen S., Bondevik G., Eberhard-Gran M., BjorvatnB.Factors associated with depressive symptoms among postnatal women in Nepal. Acta Obstet Gynecol Scand. 2007; 86(3):291-7.

[8] Patel V., Rodrigues M., DeSouza N., Gender, Poverty, and Postnatal Depression: A Study of Mothers in Goa, India. The Americal Journal of Psychiatry. Volume 159 Issue 1, January 2002, pp. 43-47.

[9] Budhathoki N., Dahal M., Bhusal S., Ojha H., Pandey S., Basnet S1. Violence against Women by their Husband and Postpartum Depression. Journal of Nepal Health Research Council, 2012 Sep;10(22):176-80
[10] Ellen Y., Cheryl A., Siobán D, Harlow, Z., Yan J., Huixia Y., Postpartum depression and traditional postpartum care in China: Role of Zuoyuezi. International Journal of Gynecology Obstetrics. March 2009Volume 104, Issue 3, Pages 209-213. [6]NDHS. Nepal Demographic and Health Survey. Preliminary Report. 2006.

[11] Budhathoki N., Dahal M., et.al. Violence against Women by their Husband and Pospartum Depression; Journal of Nepal Health Research Council. Vol.10 No 3 Issue 12 September. 2012; pp 176-180.

[12] Signe K.D. Depression and sleep in the postnatal period. A study in Nepal and Norway. Dissertation for the degree philosophiae doctor $(\mathrm{PhD})$ at the University of Bergen. 2009.

[13] Okano, T. An epidemiological and clinical investigation of postpartum psychiatric illness in Japanese mothers. Journal of Affectiv Disorders. 1998; 48, pp233-240.

[14] Deborah, A., Bridget, F., Chou, S., Frederick, S. The Impact of Partner Alcohol Problems on Women's Physical and Mental Health. The Journal of Studies on Alcohol and Drugs. 2007; vol 68: Issue 1.

[15] Satoh A., Kitamiya, C., Yaegashi, Y. Influence of Smoking on Postpartum Depression in Japan. Health Science Research. Open Access, Vol.5, No.8, 1254-1260 (2013) Retrieved Oct. 1, 2013. http://dx.doi.org/10.4236/health.2013.58170.

[16] Eysenck, H. The biological basis of personality. Springfield: CC Thomas. 1967.

[17] Gilbert, D. The psychology of the smoker. In Bollinger CT, Fagerström KO, editors. The Tobacco Epidemic. ProgRespir Res [Basel] 1997; 28: pp132-150.

[18] Baker, D., Taylor, H. The relationship between condition-specific morbidity, social support and material deprivation in pregnancy and early motherhood. ALSPAC Survey Team. Avon Longitudinal Study of Pregnancy and Childhood. Social Science Medicine 1997; 45(9):1325-36.

[19] Brugha et al. Pragmatic randomized trial of antenatal intervention to prevent post-natal depression by reducing psychosocial risk factors; Cambridge University Press. Psychological Medicine Psychological Medicine / Volume/ Issue 06 / November 2000, pp 1273-1281, 2000.

[20] Nielsen Forman D, Videbech P, Hedegaard M, Dalby Salvig J, Secher NJ. Postpartum depression: identification of women at risk. BGOJ: An International Journal of Obstetrics and Gynaecology. Bjog. 2000; 107(10):1210-7.

[21] Stern, G., \& Kruckman, L. Multi-disciplinary perspectives on post-partum depression: An anthropological critique; Social Science \& Medicine, Volume 17, Issue 15, 1983; pp 1027-1041. 